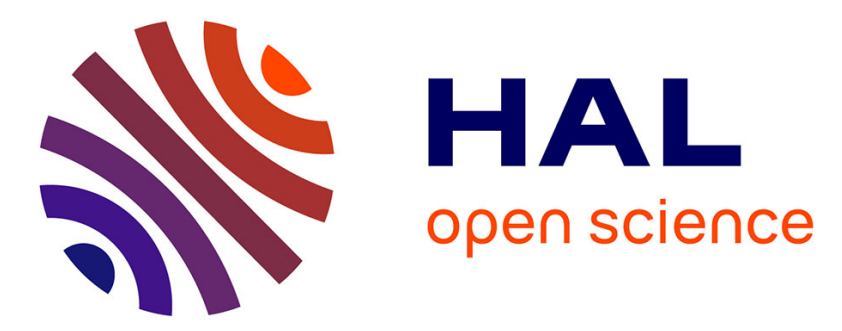

\title{
Radiative transfer sensitivity to the accuracy of canopy structure description. The case of a maize canopy
}

Marisa España, Frédéric Baret, Frank Aries, Bruno Andrieu, Michaël Chelle

\section{To cite this version:}

Marisa España, Frédéric Baret, Frank Aries, Bruno Andrieu, Michaël Chelle. Radiative transfer sensitivity to the accuracy of canopy structure description. The case of a maize canopy. Agronomie, 1999, 19 (3-4), pp.241-254. hal-00885927

\section{HAL Id: hal-00885927 \\ https://hal.science/hal-00885927}

Submitted on 1 Jan 1999

HAL is a multi-disciplinary open access archive for the deposit and dissemination of scientific research documents, whether they are published or not. The documents may come from teaching and research institutions in France or abroad, or from public or private research centers.
L'archive ouverte pluridisciplinaire HAL, est destinée au dépôt et à la diffusion de documents scientifiques de niveau recherche, publiés ou non, émanant des établissements d'enseignement et de recherche français ou étrangers, des laboratoires publics ou privés. 
Agronomie 19 (1999) 241-254

(C) Inra/Elsevier, Paris

\title{
Original article
}

\section{Radiative transfer sensitivity to the accuracy of canopy structure description. The case of a maize canopy}

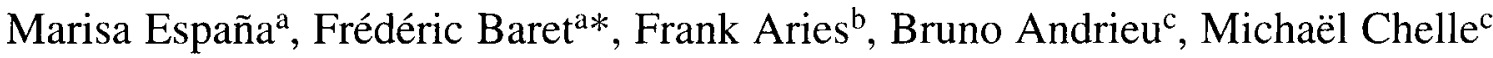 \\ ${ }^{a}$ Bioclimatologie, Inra, site Agroparc, 84914 Avignon cedex, France \\ ${ }^{\mathrm{b}}$ Biométrie, Inra, site Agroparc, 84914 Avignon cedex, France \\ ' Bioclimatologie, Inra, 78850 Thiverval-Grignon, France
}

(Received 12 November 1998; accepted 5 March 1999)

\begin{abstract}
Ray tracing techniques can be very accurate for radiative transfer computation in complex canopy architecture. However, they are limited by the large computer resources required which increase with the number of triangles used to approximate the actual canopy structure. The objective of this study was to investigate the sensitivity of the gap fraction, the leaf inclination distribution function and the bi-directional reflectance, to the detail with which canopy architecture was represented. We considered a maize canopy described using a realistic 3D architecture model. We showed that 24 plants allowed us to obtain an accurate representation of the gap fraction and the leaf angle distribution function with the finest triangulation level studied, i.e. with triangles smaller than $0.025 \mathrm{~m}$. We then degraded the canopy architecture by non-representing leaf undulation. Results showed that in our conditions, leaf undulation induced only marginal variation of the gap fraction, the leaf inclination distribution function and the bi-directional reflectance. We then finally degraded canopy architecture by dividing by a factor of 10 the number of triangles used in the previous representation. Here again, results showed marginal effects on the variables investigated. Conclusions are drawn and discussed in view of radiative transfer computation. (ㅇ Inra/Elsevier, Paris.)
\end{abstract}

maize structure / 3D architecture / radiative transfer / reflectance gap fraction / leaf inclination distribution function

Résumé - Sensibilité du transfert radiatif à la précision de la description de la structure du couvert. Le cas d'une culture de maïs. Les techniques de lancé de rayons peuvent être très précises pour calculer le transfert radiatif dans des couverts à l'architecture complexe. Toutefois, elles sont limitées par les importantes ressources informatiques qu'elles nécessitent qui augmentent avec le nombre de triangles utilisés pour donner une approximation de l'architecture du couvert. L'objectif de cette étude est d'analyser la sensibilité de la fraction de trou, de la fonction de distribution des inclinaisons foliaires et de la réflectance bi-directionnelle à la qualité de la représentation de l'architecture. Nous avons consi-

Communicated by Gérard Guyot (Avignon, France)

* Correspondence and reprints

baret@avignon.inra.fr 
déré un couvert de maïs décrit grâce à un modèle réaliste de structure 3D. Nous avons montré que 24 plantes suffisent pour représenter précisément la fraction de trou et la fonction de distribution des inclinaisons foliaires en utilisant le niveau de triangulation le plus fin, c'est-à-dire des triangles de dimension inférieure à $0,025 \mathrm{~m}$. Nous avons alors dégradé cette description en ne représentant pas le gaufrage des feuilles. Les résultats montrent que dans nos conditions, le gaufrage des feuilles n'induit que des changements marginaux sur la fraction de trou, la fonction de distribution des inclinaisons foliaires et la réflectance bi-directionelle. Nous avons enfin dégradé l'architecture en divisant par dix le nombre de triangles utilisés précédemment. Ici encore, les résultats montrent un effet marginal sur les variables considérées. Les conclusions sont tirées et discutées dans l'optique du calcul du transfert radiatif. (@ Inra/Elsevier, Paris.)

maïs / structure / architecture 3D / transfert radiatif / réflectance / fraction de trou / fonction de distribution des inclinaisons foliaires

\section{Introduction}

Radiative transfer computation is widely used for a large range of applications, including remote sensing, $[12,14,16]$ and canopy functioning studies [1]. Different types of radiative transfer model were developed. They are mainly characterised by the way in which they represent canopy architecture. They range from the simplest ones where canopies are assumed to be turbid medium [10, 13, 15], up to the more realistic ones where canopy architecture is explicitly described in its complexity $[3,8,9,11]$. Ray tracing techniques allow us to compute very accurately the radiative transfer if enough rays are considered. They have been recently developed thanks to the increase in computing capacity. Concurrently, realistic 3D canopy architecture models have been proposed $[5,7,11]$. Although the use of ray tracing radiative transfer models associated with a detailed description of canopy architecture is very attractive, they are still demanding in terms of computer resources and time. Therefore, they are not currently used operationally.

Ray tracing techniques consider the canopy as a set of elementary geometrical primitives. The radiative transfer resumes the computation of the intersection of a line (the ray) with the elementary primitive. In the case of intersection, the direction and intensity of reflected and transmitted rays are drawn within specified distribution functions. In the case of high order rational parametric surfaces, the problem may be very complex [2]. The triangle is the simplest primitive for which the intersection with a line is straight forward and is widely used. The computation time associated with ray tracing techniques applied to canopies described by triangles depends mainly on the number of triangles and on the number of rays $(n)$. The accuracy of the radiative transfer computation evaluated with the RMSE varies as $1 / \sqrt{ } n$. The number of rays used is, therefore, chosen so as to obtain the desired accuracy. In consequence, the accuracy of the radiative transfer variables as compared to that of actual canopies will depend mainly on the number of triangles used to describe the actual surfaces of canopy elements. However, the computation time and computer resources required for radiative transfer computation although not proportional to the number of triangles, grows significantly with the number of triangles. On the other hand, the amount of memory required increases significantly with the number of geometric primitives. A compromise between accuracy and computer time and resources should be found to optimise the efficiency of such radiative transfer techniques.

The objective of this study is to evaluate the sensitivity of radiative transfer simulation to the accuracy of canopy structure description, i.e. the number of triangles used to describe canopy surfaces. We will consider maize canopies which have a relatively simple structure. The reference maize canopy architecture used is that developed by España et al. [7] which describes the leaf shape, curvature and possible undulation very realistically using sophisticated parametric surfaces. The number of triangles considered to describe a given 
maize field at a given development stage is the product between the number of plants used to represent the variability between plants and the average number of triangles necessary to approximate the actual surfaces of a plant. We will investigate both aspects using the following three type of variables involved in radiative transfer processes:

1) the gap fraction ( $\mathrm{Po}$ ), which determines the penetration of light in the canopy, and is, therefore, one of the main variable governing the radiative transfer;

2) the leaf inclination distribution function (LIDF), used to compute the amount and directionality of the photons that are absorbed or scattered by the leaves;

3) the bi-directional reflectance distribution function (BRDF), which is the output of radiative transfer models used for remote sensing applications.

We will first describe the method developed to compute these three variables, along with the triangulation procedure used to approximate the parametric surfaces of the original canopy architecture model of España et al. [7]. We will then evaluate the number of plants used to obtain representative values of canopy radiative transfer variables for the best triangulation level considered. We will finally analyse the sensitivity of the accuracy of the radiative transfer computation to the triangulation level.

\section{Methods}

We will first present the 3D maize canopy architecture and the triangulation procedure used. We will then describe how the variables of interest (gap fraction, leaf angle distribution and bi-directional reflectance) were computed.

\subsection{The 3D canopy architecture model}

We used the 3D canopy architecture model proposed by España et al. [7] to describe maize plants at the anthesis stage. It is based on simple parametric mathematical expressions to represent leaf and stem surfaces. It allows us to realistically describe the shape, curvature and undulation of the leaves in the plant. The parameters of the leaf model are randomly drawn within distribution laws that were calibrated over experimental observations [6]. The number of leaves, size, position and height of the plant were also randomly drawn according to experimental observations. The loss and senescence of leaves were not considered here, i.e. all the leaves produced were still present. The sowing pattern of the canopy was $0.7 \mathrm{~m}$ between rows and $0.2 \mathrm{~m}$ between plants on the row. The average height was 1.8 $\mathrm{m}$, with 17 leaves representing a leaf area index of 3.6.

\subsection{The triangulation procedure}

The stems were described by cone frustums. Their triangulation scheme was fixed in this study and made of 500 triangles [6]. Leaf triangulation was achieved in two steps: the leaf was initially divided into 20 triangles organised in a pattern that respect the mid-rib axis. Then, this initial triangulation was refined progressively by dividing by two the longest side of each triangle if its length was longer than a given threshold value. For the initial triangulation, the length of the longest side of the triangles was $0.2 \mathrm{~m}$. The finest triangulation level compatible with a reasonable computation time was achieved with a maximum length of the longest side of the triangles lower than $0.025 \mathrm{~m}$. We demonstrated that this procedure provided convergence for the area, the distance and the normal of the surface: when the threshold value tends toward zero, the distance between the triangulated surface and the actual parametric surface tends toward zero, the orientation of the normal tends to the actual distribution, and the area converges to the finite value of the actual surface.

\subsection{Computation of the variables of interest}

We will describe here the procedure used to compute the variables over which the accuracy of the radiative transfer will be evaluated. This will concern the gap fraction, the leaf inclination distribution and the bidirectional reflectance.

\subsubsection{The gap fraction: the X-ray method}

A method was developed here to compute the gap fraction map of a canopy with given sowing pattern but for which the plant features and the azimuth orientation are random. Because of the similarity between the gap fraction maps obtained and X-ray images, we called the method the 'X-ray method'. It splits the computation of the gap fraction into two consecutive steps corresponding to the plant and the field levels. It will therefore be 
easier to extend the results from the plant level to the canopy level for a range of sowing patterns. This method assumed that the plants are independent of each other, and that their azimuths are randomly distributed. These assumptions are obviously wrong for actual canopies in which strong interactions between plants through competition for space and resources play a dominant role. However, due to the complexity of these processes and the consequence on canopy architecture, we decided to not take them into account. Nevertheless, the images of the canopies simulated gave quite realistic impressions of actual maize canopy structure [7].

The canopy is made of $m$ plants, each one being drawn randomly within a set of $q$ plants available. The gap fraction $P o_{\text {canopy }}(\Omega)$ of the canopy is the average on the projection plane (a plane perpendicular to direction W) of the canopy gap fraction $P o_{\text {canopy }}(\Omega,[x, y])$ corresponding to each point $[x, y]$ on the projection plane. Because the plants were assumed independent, the canopy gap fraction $P o_{\text {canopy }}(\Omega,[x, y])$ for point $[x, y]$ and direction $\mathrm{W}$ can be computed from the plant gap fraction $P o_{p l a n t}(\Omega,[x, y], j, \Phi)$ where $i(1<i<m)$ represents the index of the plant referring to the plant position in the canopy, and the index $j(1<j<q)$ refers to a plant individual chosen from the $q$ available plants for each plant position [ $i]$, and $\Phi$ is the azimuth of the plant:

$$
P o_{\text {camopv }}(\Omega,|x, y|)=\prod_{1}^{m} P o_{\text {plant }|i|}(\Omega,|x, y|, j, \Phi)
$$

Note that at this stage $P o_{\text {plant }}(\Omega,[x, y], j, \Phi)$ takes only binary values 0 or 1 .

For a plant placed in a given position in the canopy $[i]$, and for which the features (described by the choice of the plant $j$ ) and azimuth orientation $\Phi$ are unknown, the corresponding average gap fraction value

$\overline{P o_{\text {plan } \mid i]}(\Omega,|x, y|)}$ computed over the $q$ possible plants available and all the azimuth orientations by $1^{\circ}$ step is:

$$
\overline{P o_{\text {plam } \mid \text { | } \mid}(\Omega,|x, y|)}=\frac{1}{q} \sum_{j=1}^{q} \frac{1}{360} \sum_{\Phi=1}^{360} P o_{\text {ptaml }|i|}(\Omega,|x, y|, j, \Phi)
$$

Figure 1 presents the gap fraction maps of a single plant averaged over the $360^{\circ}$ azimuth angles for three particular directions. The average gap fraction computed following equation (2) on a set of $q$ plants resulted in the average plant gap fraction maps (figure 2). The plant gap fraction maps are symmetrical with regards to the stem axis, because of the random plant azimuth position. The gap fraction increases from zero for the stem, up to unity when reaching the external envelope of the gap fraction maps defined by the tips of the more external leaves. The pattern of the envelope is very smooth for nadir $\left(0^{\circ}\right)$ gap fraction, because it is defined by the rotation of the more extended leaf using $1^{\circ}$ azimuth steps. Conversely for the horizontal $\left(90^{\circ}\right)$ gap fraction the irregular envelope pattern is explained by the variability observed from plant to plant of the length, insertion height and curvature of the leaves. The pattern of the $45^{\circ}$ gap fraction envelope is intermediate between the horizontal and vertical situations.

The average canopy gap fraction for direction $\mathrm{W}$ and point $[x, y]$ is derived from the average plant gap fraction:

$$
\overline{P o_{\text {cunopy }}(\Omega,|x, y|)}=\prod_{i=1}^{m} \overline{P o_{\text {plunt }[i]}(\Omega,|x, y|)}
$$

\subsubsection{The leaf inclination distribution function}

Because the azimuths of the plants were assumed to be randomly distributed, we will only consider the distribution of the zenith angles.

The leaf inclination distribution function of the actual canopy was derived from the distribution of the direction of the normals of the parametric surface. Because of the complexity of the parametric surface, this cannot be analytically derived in the general case. However, it is possible to compute the directions of any single normal on the parametric surface (figure 3). The distribution of the directions of the normals can, therefore, be approximated by sampling sufficiently the parametric surface. This was achieved using a fine triangulation and considering the corners and the centre of each triangle represented in the $3 \mathrm{D}$ space. In this case, for each point of the surface sampled, the direction of the normal was weighted by the local area around it, approximated by the area of the associated sub-triangles as shown in figure 3.

The distribution of the normals of the triangulated surface was computed as the distribution of the direction of the normals of the triangles weighted by their area. A very good agreement was found between the LIDF computed over the parametric surface and that obtained over the triangulated surface for the finest triangulation level. In the following, the LIDF will always be computed over the triangulated surface.

\subsubsection{The bi-directional reflectance distribution function}

We used the PARCINOPY ray tracing model developed by Chelle [3]. This model allows us to simulate 

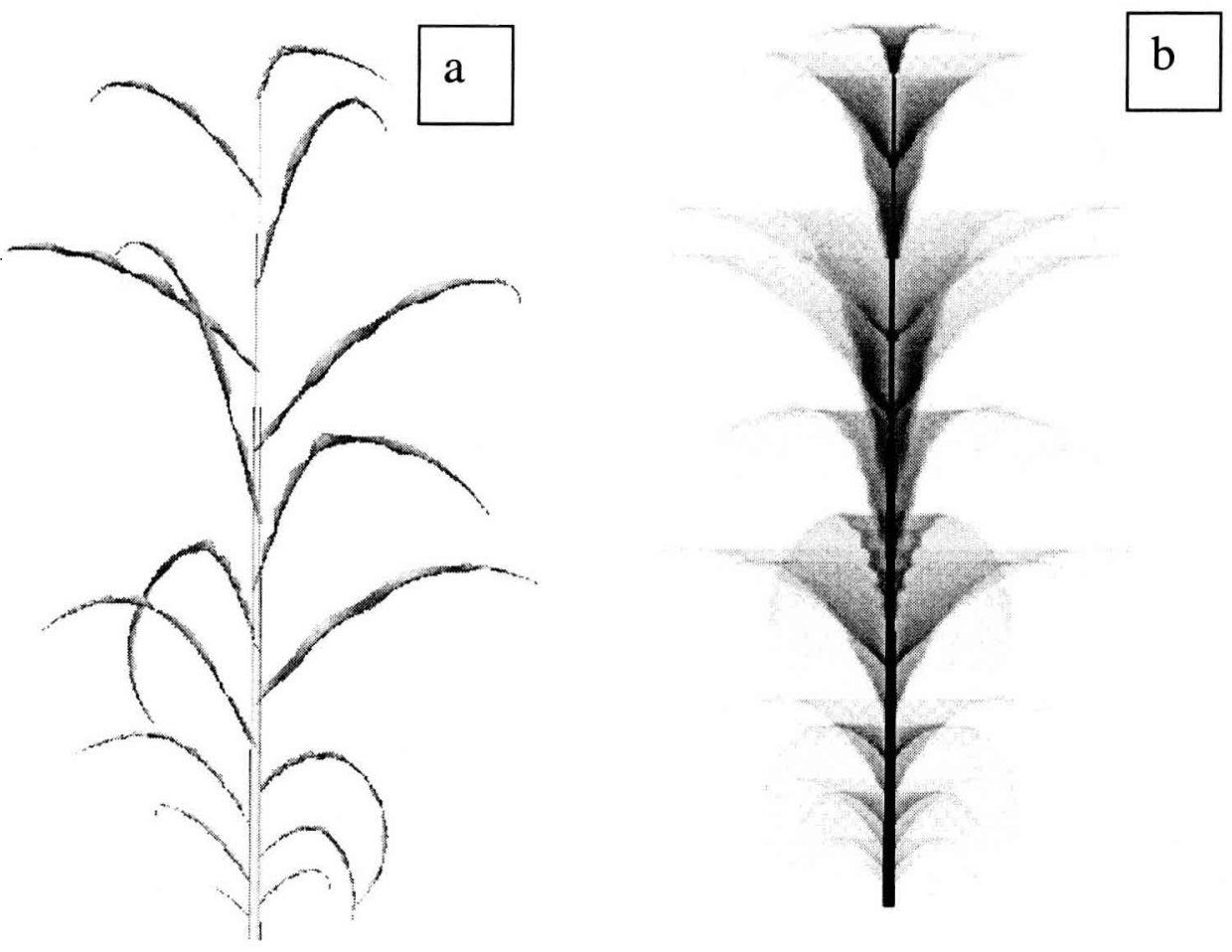

$\mathrm{b}$

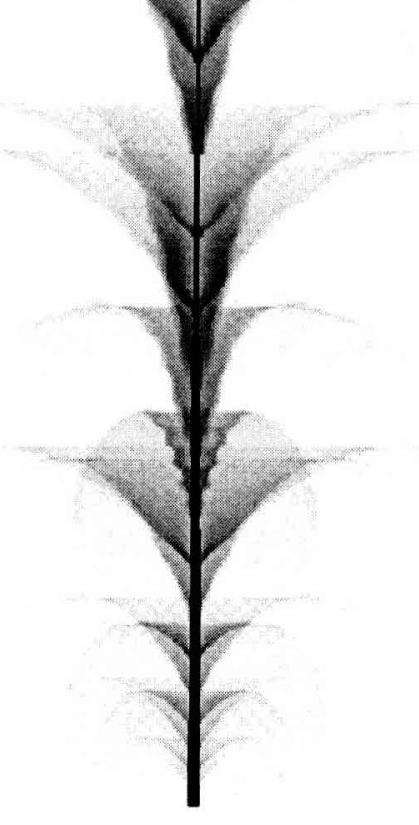

C

d

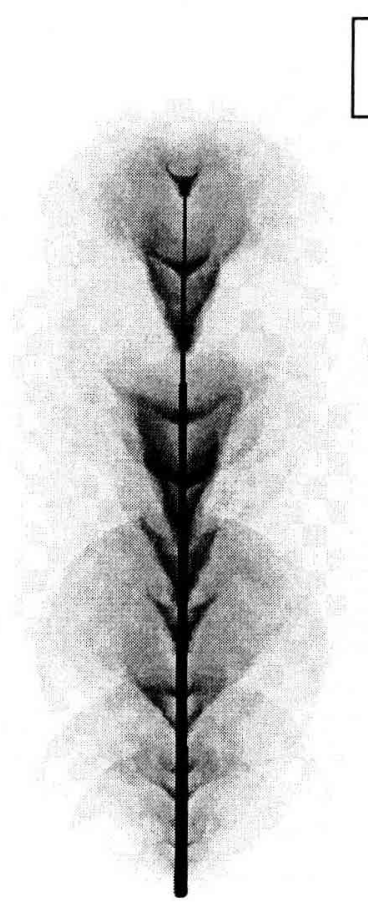

Figure 1. Representation of an adult maize plant (a) and the corresponding gap fraction maps obtained for $90^{\circ}$ (b), $45^{\circ}$ (c) and $0^{\circ}$ (d) directions by averaging the gap fraction over the 360 azimuth positions. Black correspond to $P o=0$ and white to $P o=1$. 

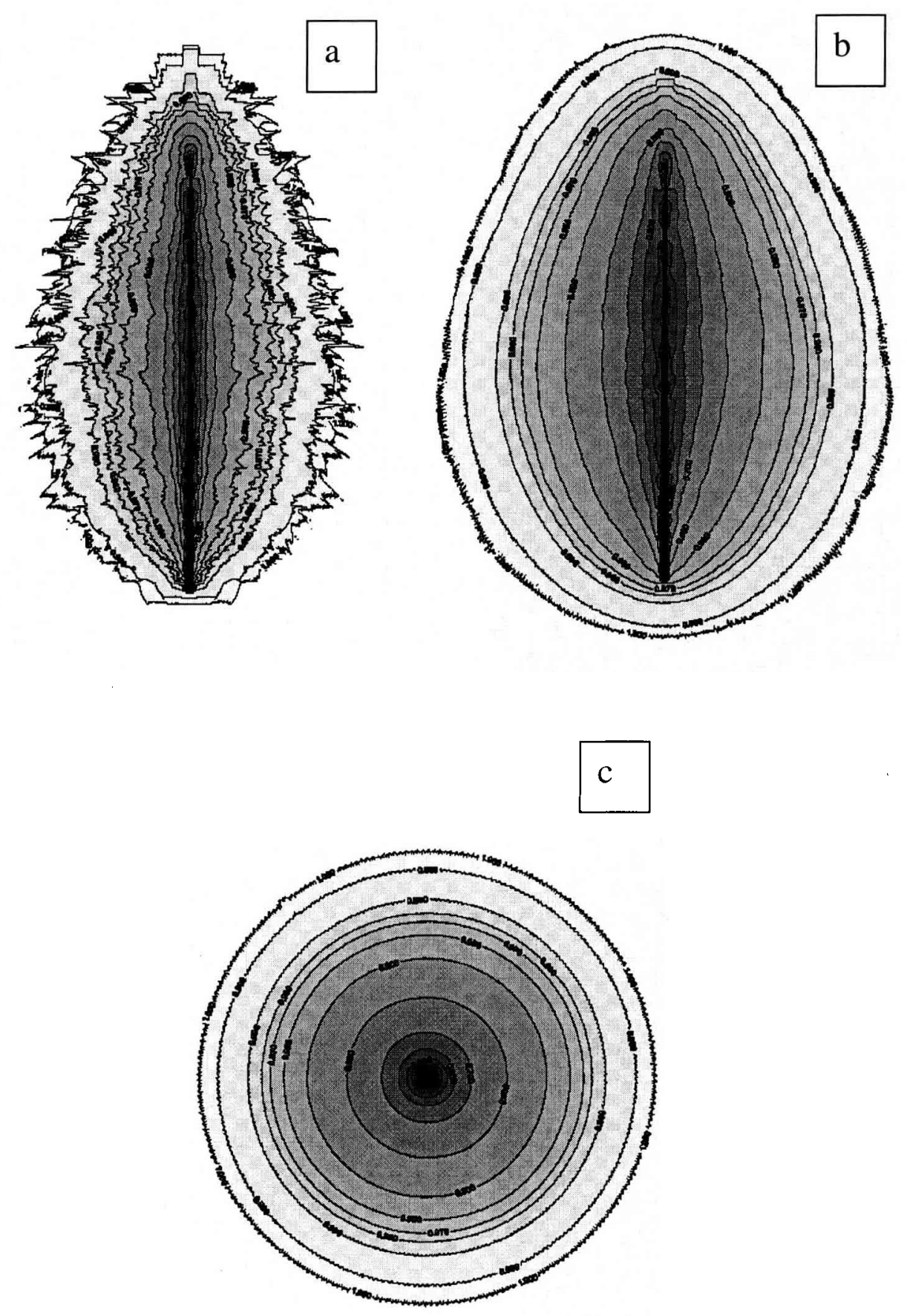

Figure 2. Gap fraction maps averaged over 120 plants for $90^{\circ}$ (a), $45^{\circ}$ (b) and $0^{\circ}$ (c) zenith directions. Black corresponds to Po $=0$ and white to $P_{0}=1.0$. 


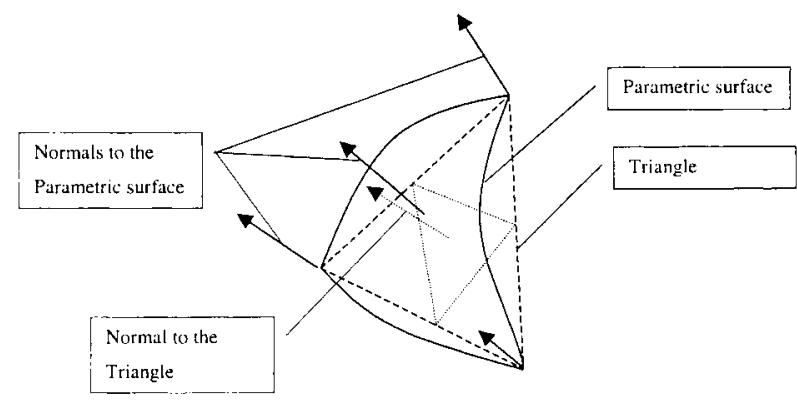

Figure 3. Schematic representation of how the normals to the parametric surface and that corresponding to the triangle were computed.

infinitely extended canopies by replicating to infinity an elementary cell. The rays were thrown from the source position in a given direction. They had an initial weight of 1.0. When intercepted by a canopy element, the weight and the direction of the scattering were modified according to the incidence direction and to the optical properties of the element considered. The iterative process was stopped when the weight of the ray tended toward zero, or when it escaped the canopy. In this case it contributed to the reflectance in the escape direction. The view directions were discretised into $5^{\circ}$ solid angles. Around the hot spot $2^{\circ}$ solid angles were used to better describe this local feature.

We restricted our study to the principal plane between $-85^{\circ}$ and $+85^{\circ}$ zenith angles where most of the directional variation was observed. The sun was illuminating the scene from a $45^{\circ}$ zenith angle with a black sky. We considered red $(670 \mathrm{~nm})$ and near infrared bands $(850 \mathrm{~nm})$ for which leaf reflectance were 0.06 and 0.44 , and leaf transmittance 0.03 and 0.47 , respectively. The soil reflectance was set to 0.20 for both wavelengths. Leaf reflectance and transmittance, as well as soil reflectance were assumed to be lambertian. Three million rays were thrown providing a relative accuracy around $2.5 \%$.

\section{Number of plants for accurate radia- tive transfer computation}

In this section, we investigate the sensitivity of the gap fraction and the leaf inclination distribution function to the number of plants used to build the
$3 \mathrm{D}$ canopy scene. For this purpose, we used the best description of each plant using the finest triangulation level considered $(0.025 \mathrm{~m})$.

The experience acquired in the measurement of reflectance over maize canopies showed that a minimum of a few square meters of the canopy should be sampled to obtain a representative value, i.e. with a relative accuracy better than $10 \%$ [4]. In our case, we considered a sample of 24 plants corresponding to a $3.36 \mathrm{~m}^{2}$ area according to the plant density used ( $\left.7.14 \mathrm{plt} . \mathrm{m}^{-2}\right)$. It corresponds to three rows of eight plants. We analysed the variability of the gap fraction and that of the leaf inclination distribution between five replicates of samples made up of 24 plants. This limited sample size is justified by the important computer resources required for the generation of the 120 plants.

\subsection{Gap fraction}

We estimated the gap fraction using the X-ray method as described earlier allowing us to de-couple the plant from the canopy levels. We will therefore study the plant level, and then investigate how the results transfer to the canopy level. We considered three zenith directions: $0^{\circ}$ (nadir), $45^{\circ}$ and $90^{\circ}$ (horizontal viewing).

\subsubsection{Plant level}

We computed the gap fraction maps for each of the five samples of 24 plants and for each of the three zenith directions. These gap fraction maps were compared to that obtained using the five samples of 24 plants, i.e. 120 plants (figure 2). For each pixel of the plant gap fraction maps and for each of the five samples of 24 plants, we calculated the difference with the 120 plant average value. Results show that the distribution of this difference for the three directions considered is centred on zero where the frequency was maximum (figure 4). More than $99 \%$ of the pixels of each of the five samples of 24 plants are associated with a difference smaller than \pm 0.02 . The largest differences are observed close to the envelope of the gap fraction maps, where the variability between plants is maximum because the number of leaves involved 
in the gap fraction computation is the least. For the same reason, the variability associated to the vertical direction is the highest. We should note here that the highest variability is therefore observed for the highest values of the gap fraction maps, indicating the potential interest of using relative errors.

Because of the small differences observed on the average plant gap fraction maps between the five samples of 24 plants, we concluded that a group of 24 plants is sufficient to estimate the average gap fraction map of the plant within a very good accuracy when all the possible azimuth positions of the plants are considered.

\subsubsection{Canopy level}

For a direction $\Omega(\theta, \phi), \theta$ and $\phi$ being the zenith and azimuth angles relative to the row, respectively, simple geometric considerations allowed us to derive the canopy gap fraction from the plant average gap fraction maps. As seen earlier, because the plants were considered to be independent from each other, the gap fraction at the canopy level for a direction $\Omega$ and point $[x, y]$ on the projection plane is the product of the plant gap fraction values for the direction $\Omega$ and point $[x, y]$ considered (equation (3)). The uncertainty in the plant gap fraction $\left(\triangle P o_{\text {plant }}\right)$ will therefore be transferred up to the canopy level ( $\left.\Delta P O_{\text {canopy }}\right)$ depending on the number of plants $m$ in the optical path for direction
$\Omega$ and their average gap fraction value $P o_{\text {plant }}$. We illustrated this problem using a simple case where the direction $\Omega$ considered is horizontal and parallel to the rows. If we assume that the rows are separated enough so that the envelopes of the plant gap fraction maps do not overlap between rows, the gap fraction map of the canopy in this condition is the average plant gap fraction map at the power $m, m$ being the number of plants considered in the row (the optical depth). The error at the canopy level $\triangle P O_{\text {canopy }}$ derived from that at the plant level $\triangle P o_{\text {plant }}$ according to:

$$
\Delta \mathrm{P} o_{\text {canopy }}=\left(P o_{\text {plant }}+\Delta P o_{\text {plant }}\right)^{m}-P o_{\text {plant }}{ }^{m}
$$

Figure 5 shows that for the usual range of plant gap fraction relative errors $\left(-0.02<\Delta P o_{\text {plant }}<+0.02\right)$, the errors at the canopy level remains small except for values of $P o_{\text {plant }}$ higher than 0.8 . For the highest optical depths, it can reach large values. This corresponds to the large optical depths considered $(m=20)$. For zenith angles lower than $60^{\circ}$ the optical path will always be lower than $m=20$. The highest levels of error at the canopy level will therefore be concentrated for the highest zenith angles and at the edges of the gap fraction maps where the gap fraction values are close to 1.0.

The error at the canopy level as a function of the error at the plant level is almost symmetrical with respect to the origin $\left(\Delta P o_{\text {plant }}=0\right)$ for this range of
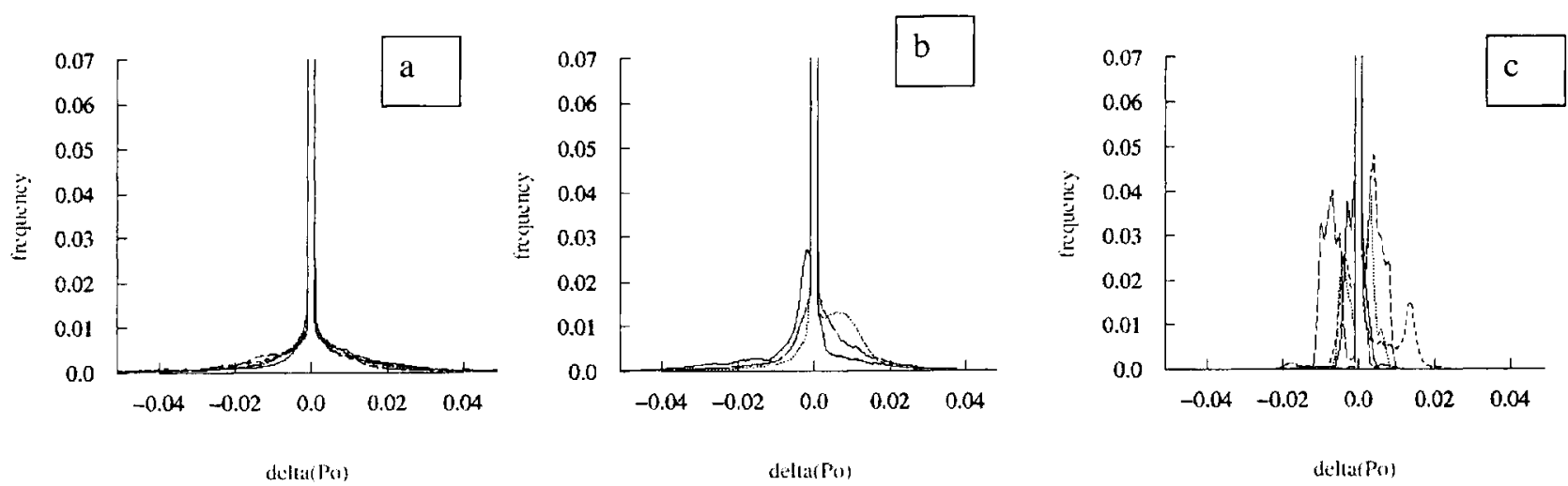

Figure 4. Distribution of the difference between the gap fraction (delta(Po)) averaged over five groups of 24 plants and that averaged over 120 plants. (a) $90^{\circ}$, (b) $45^{\circ}$, (c) $0^{\circ}$ zenith directions. 


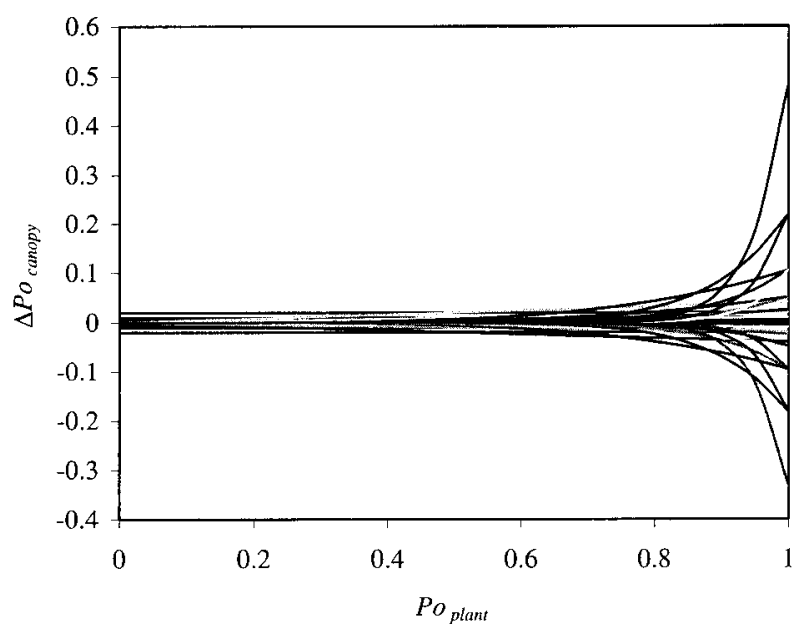

Figure 5. Variation of the error on canopy gap fraction $\left(\triangle P o_{\text {canopy }}\right)$ as a function of the plant gap function $\left(P o_{\text {plant }}\right)$ for a range of optical depths $(m=1,2,5,10,20)$ and values of plant gap fraction errors $\left(\Delta P o_{\text {plant }}=-0.020,-0.010,-0.005\right.$, $0.000,0.005,0.010,0.020$ ).

plant errors and optical paths (figure 5). This will induce a symmetry in the distribution of the errors of gap fraction at the canopy level because the distribution of the plant errors $\left(\Delta P o_{\text {plant }}\right)$ was symmetrical with regards to the zero value (figure 4 ).

We conclude that the average gap fraction at the canopy level will generally be associated with small errors in any direction $\Omega$, if computed with a gap fraction map accurate enough. Therefore, as shown in the previous section, gap fraction maps computed over a sample of $q=24$ plants will provide results with a good accuracy.

\subsection{Leaf inclination distribution function}

We computed the leaf inclination distribution functions individually for the five samples of 24 plants. The same was carried out for the 120 plants that provided a quite smooth LIDF (figure $6 a$ ) which partly confirmed that 120 plants were sufficient to obtain a representative description of the LIDF at the canopy level. The computation of the LIDF used $5^{\circ}$ width classes.

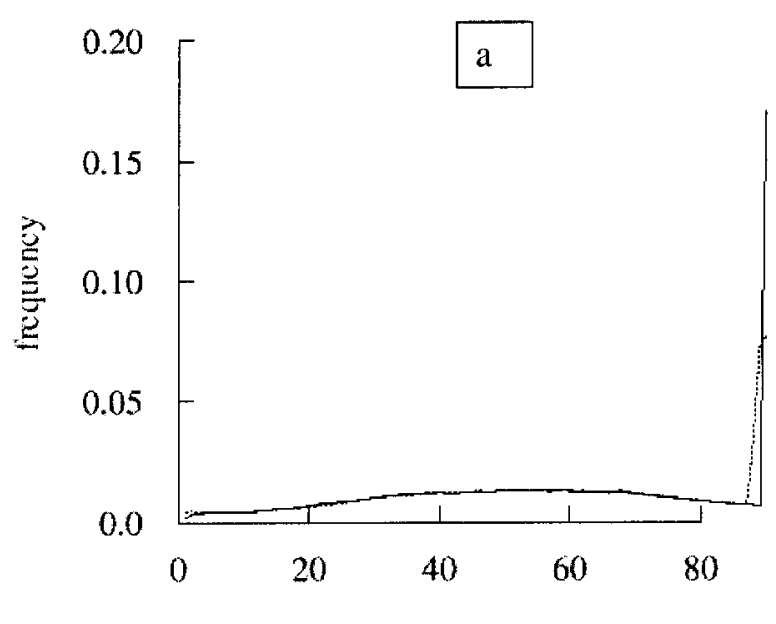

inclination angle( $\left.{ }^{\circ}\right)$

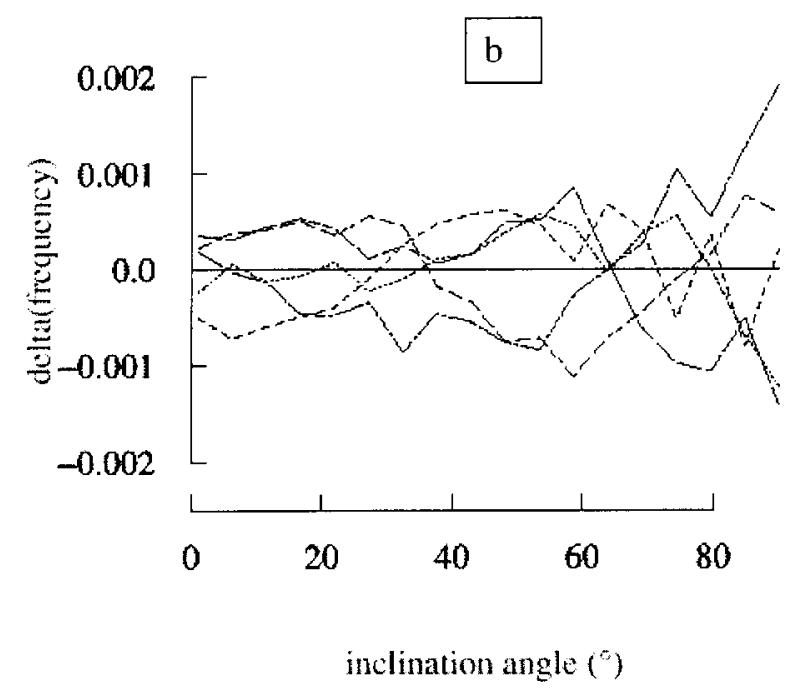

Figure 6. Leaf inclination distribution (frequency) computed over 120 plants (a). Difference in the distribution (delta(frequency)) observed for five groups of 24 plants as compared to that observed over the 120 plants (b).

Results show that the maximum difference between each of these five LIDF and that calculated on the 120 plants for each of the $5^{\circ}$ classes are lower than 0.002 . The average difference is close to 0.0005 corresponding to a relative error value better than $10 \%$ on each of the $5^{\circ}$ classes (figure $6 b$ ). 
We thus concluded that 24 plants provide a representative LIDF. However, the errors could be significant in the case of the computation of the BRDF when using particular leaf phase functions such as specular ones, which could be very sensitive to the LIDF in the specular direction.

These results showed that a sample of 24 plants provides a good representation of the maize field while retaining reasonable computer resource requirements. This was achieved using the best triangulation level. In the following, we will use this 24 plants sample to investigate the sensitivity of radiative transfer variables to the triangulation level.

\section{Sensitivity to the triangulation level}

We will now evaluate the sensitivity of radiative transfer variables to the quality of the representation of the 3D architecture model, mainly driven by the triangulation level. We will compare the finest triangulation level $(0.025 \mathrm{~m})$, noted $\mathrm{T} 0$, which was considered as the reference, to two different levels of canopy structure description:

$\mathrm{T} 1$ : the same triangulation level $(0.025 \mathrm{~m})$ as $\mathrm{T} 0$ is used, but the leaf undulation is not represented. This will allow us to study separately the effect of the leaf undulation;

T2: the initial triangulation is used, which corresponded to the simplest case. We should note here that a very degraded triangulation as in the initial triangulation level did not allow us to represent the undulation of the leaves.

Figure 7 illustrates the levels of canopy architecture representation investigated.

\subsection{Gap fraction}

In the following we will focus on the plant level. We showed in the previous section how to transfer the error from the plant level to the level of the canopy using the X-ray method.
We computed the plant average gap fraction maps of a set of 24 plants with the finest triangulation level as described previously. Similarly, the plant average gap fraction maps were computed with the same set of 24 plants for the two degraded representations ( $\mathrm{T} 1$ and $\mathrm{T} 2$ ) and the three directions considered $\left(0^{\circ}, 45^{\circ}\right.$ and $\left.90^{\circ}\right)$.

The gap fraction maps of the difference between T0 plant average gap fraction with that of T1 and $\mathrm{T} 2$ were generated. The associated distribution of the error was calculated (figure 8). Results showed that the distribution of this difference for the three directions considered is almost centred on zero where the frequency is maximum: more than $99 \%$ of the pixels of $\mathrm{T} 1$ and $\mathrm{T} 2$ are associated with a difference lower than 0.02 in absolute value as compared to T0. The errors induced by the degradation of the quality of the representation of the plant due either to the absence of undulation (T1) or to the reduction in the number of the triangles (T2) are thus only marginal and of the same order of magnitude as the one due to the variability with the number of plants (figure 4). Therefore, the effect at the canopy level will also be very small, similarly to what was seen in section 3.1 .

\subsection{Leaf inclination distribution function}

The LIDF were computed over the three cases $\mathrm{T} 0, \mathrm{~T} 1$ and $\mathrm{T} 2$. The difference between $\mathrm{T} 0$ and $\mathrm{T} 1$ or T2 shows (figure 9) that the errors on the frequency are generally lower than 0.005 . The main effect of the degradation of the structure representation is due to the undulation which increased the amount of almost horizontal surfaces. The other important effect is due to the reduction of the number of triangles explaining the more noisy LIDF observed for $\mathrm{T} 2$. There were about ten times fewer triangles for the most degraded triangulation (T2 = 20200 triangles) as compared to that of the finest one (T0 $=210000$ triangles). We observed that the effect of the undulation and the triangulation level is larger than the variation induced by the number of plants used to represent the canopy (figure 6). Therefore, the undulation of the leaf and the triangulation level could induce significant 


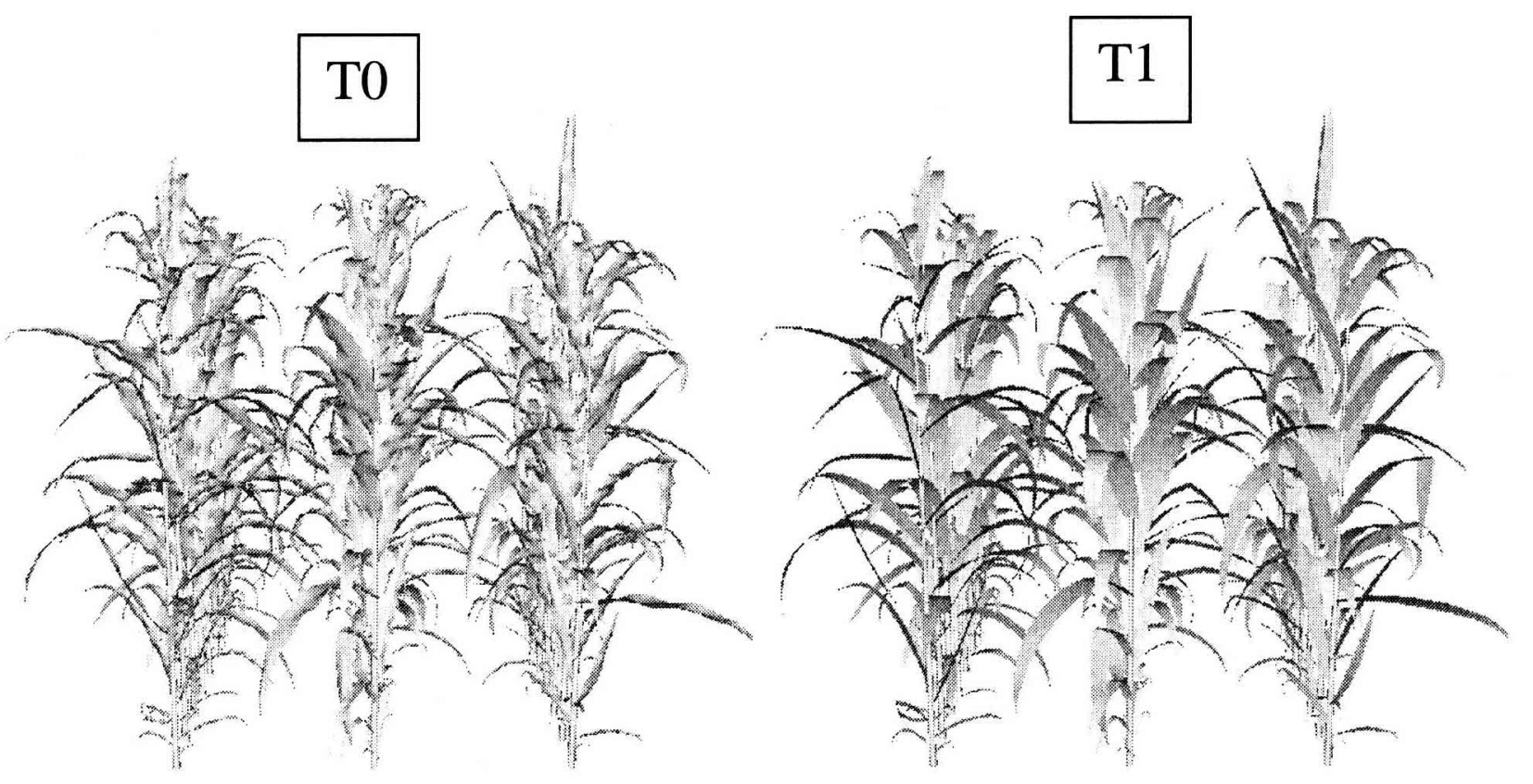

\section{$\mathrm{T} 2$}

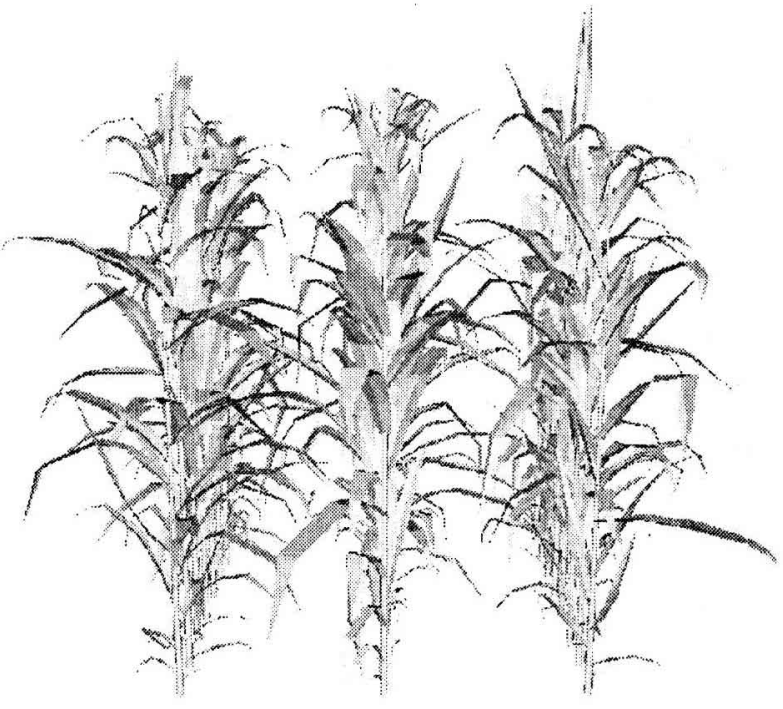

Figure 7. Representation of the maize canopy using 24 plants. The three cases considered are displayed: $T 0$ finest triangulation level and leaf undulating (210 486 triangles); T1 finest triangulation level but no leaf undulating (209 730 triangles); T2 largest triangulation level, no leaf undulating (20 200 triangles). 

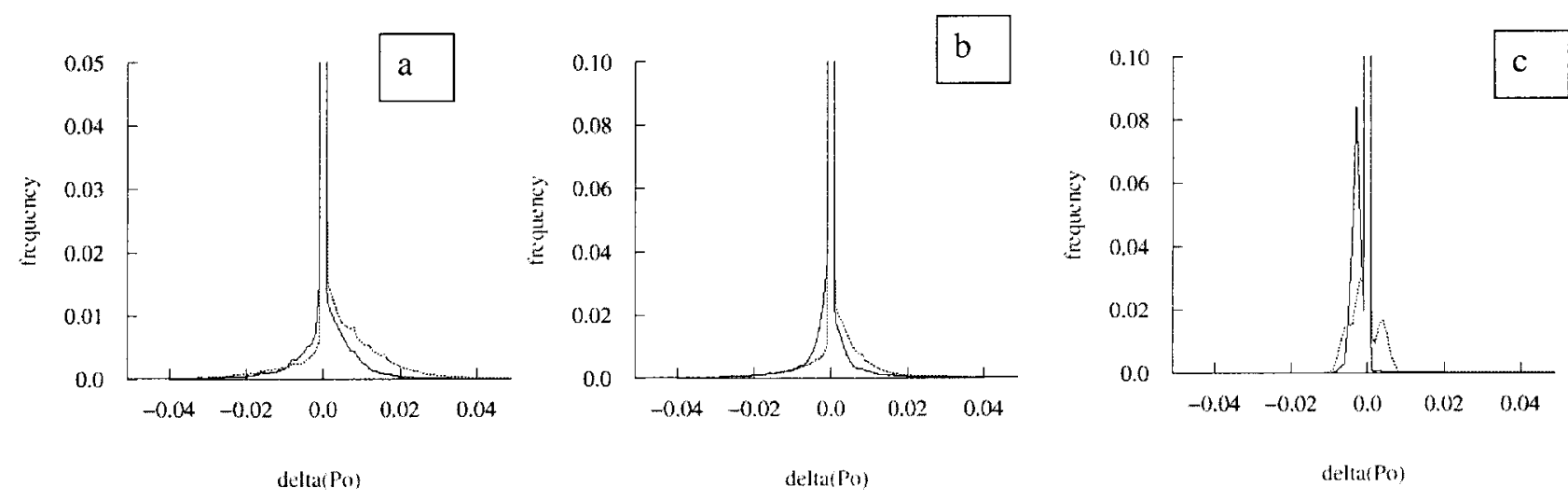

Figure 8. Distribution of the difference of gap fraction values (delta(Po)) computed for the same set of 24 plants between the reference case corresponding to the finest triangulation and undulating leaves (T0), and the plants without undulation (T1: solid line), and the plants with the simplest representation (T2: dashed line). (a) $90^{\circ}$, (b) $45^{\circ}$, (c) $0^{\circ}$ zenith directions.

changes on the LIDF when looking at the specular direction for specular leaves.

\subsection{Bi-directional reflectance distribution function}

The same set of 24 plants was used to compute the BRDF. Conversely to what was investigated previously for the gap fraction with the X-ray method, for the ray tracing simulation the plants were assigned to a single azimuth position drawn within random distributions. We verified that assigning each plant to a given random azimuth did not induce significant variation when compared to the situation where all 360 azimuth positions were explicitly considered.

Figure 10 shows the reflectance values in the red and near infrared wavebands for the best triangulation level with leaf undulation (T0). The curves are relatively smooth, confirming that the number of plants and triangles and the number of rays were large enough. The hot spot maximum peaked in the direction of the sun at $45^{\circ}$ view zenith angle. A minimum reflectance value was observed in the near infrared for nadir viewing because the fraction of the soil seen is maximum and soil reflectance is much lower than that of the leaf.

The comparison between the reference triangulation level with leaf undulation (T0) and the same triangulation level without leaf undulation (T1) does not exhibit significant differences (figure 11a). The range of variation is within the \pm 0.025 relative accuracy of the computation owing to the number of rays used. We thus concluded that in these conditions, the undulation does not significantly affect the reflectance of the canopy. Conversely, when the triangulation level was degraded up to the lowest triangulation level (T2), some slight difference appears (figure $11 \mathrm{~b}$ ). Reflectance in the red increases by $2-3 \%$ (relative values) while a relative decrease of $4-5 \%$ is observed in the near infrared. A closer inspection of the sources of variation shows that the increase in the red was due to an increase of the single scattering, the multiple scattering being almost insignificant. Conversely, the decrease in the near infrared is mainly due to a decrease in the multiple scattering, the single scattering showing no particular trend as compared to that of T0. Explanations of these subtle but significant trends were questionable and no single argument investigated led to a plausible solution.

\section{Conclusion}

This study demonstrated that the quality of canopy architecture for the estimation of radiative 


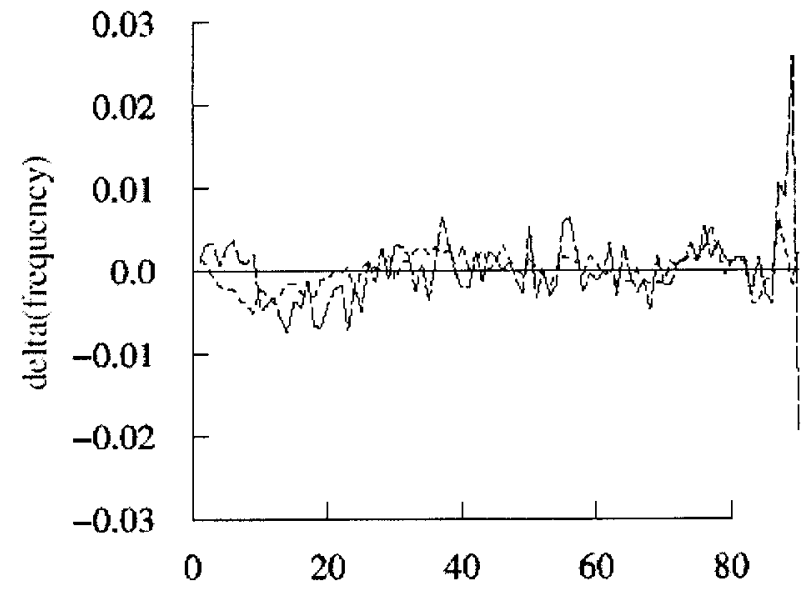

inclination angle (")

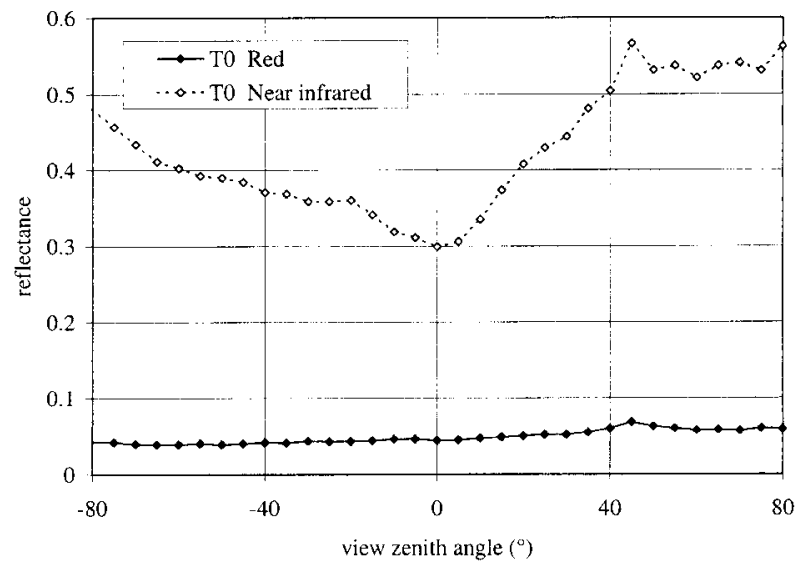

Figure 10. Reflectance computed using PARCINOPY on T0 using 24 plants in the red and near infrared domains.

ular leaf types such as needles. We should look for more general criteria that would define the pertinent level of canopy architecture description required for accurate radiative transfer computation.

However, within the range of variation of canopy architecture considered in this study, the degree of detail of the 3D architecture of the most degraded representation still allowed us to identify easily a maize canopy (figure 7 ). We should push the range of investigation to even more simple architecture, up to the simplest one corresponding to the turbid medium. It would therefore be possible to evaluate the right level of canopy architecture for a given species, and the associated radiative transfer models.

This study clearly demonstrated the potential of such an approach allowing us to manipulate easily canopy architecture, and to observe the corresponding sensitivity of radiative transfer variables. It should shift the emphasis currently put on the calculation of the radiative transfer, towards future investigation on the pertinent level of canopy architecture description.

Acknowledgements: This study was partly funded by the European Commission through the grant provided to M. España and funds coming from the Programme National de Télédétection Spatiale in France. We thank also very much $\mathrm{P}$. Clastre for his assistance in computation. They have to be confirmed over other types of canopies for which these conclusions might be different. This could be the case of species with partic- 

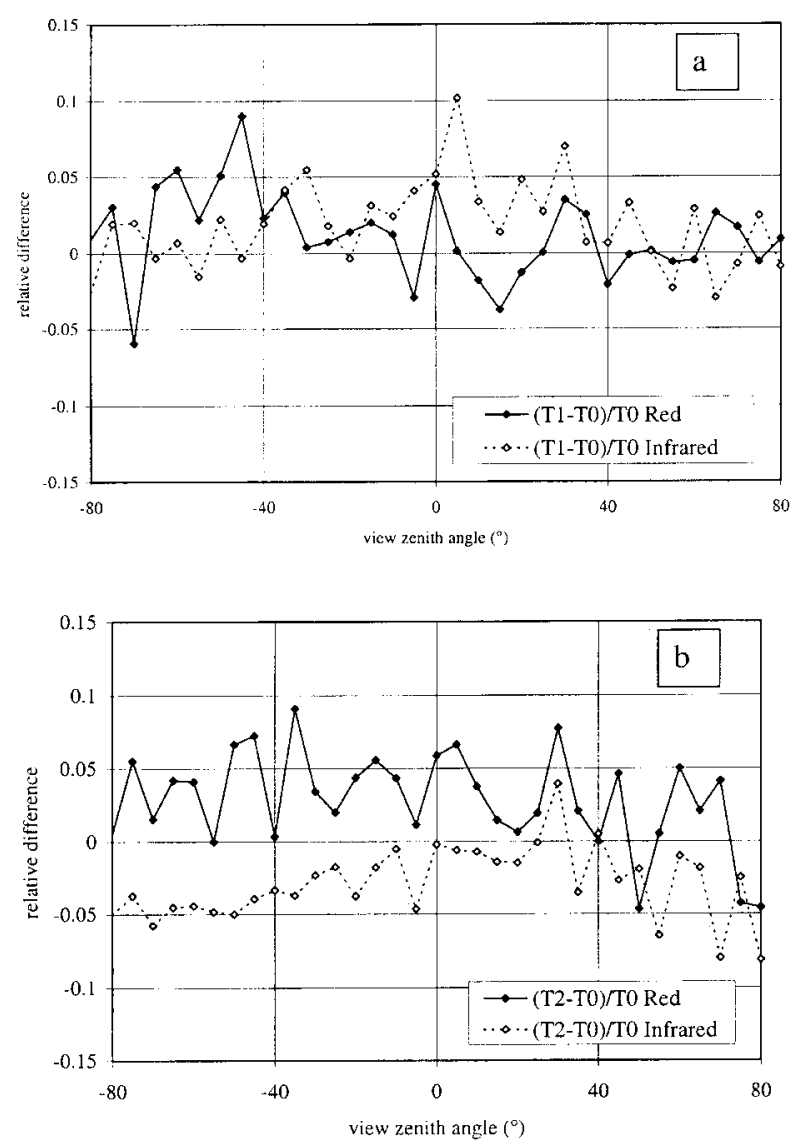

Figure 11. Relative difference computed on the reflectance in the red and near infrared domains between $\mathrm{T} 0$ and $\mathrm{T} 1$ (a), and $\mathrm{T} 0$ and $\mathrm{T} 2$ (b).

\section{References}

[1] Andrieu B., Chelle M., Clastre P., Sohbi, Y., Modélisation des échanges radiatifs à partir de maquettes informatiques, in: Cruiziat P., Lagouarde J.P. (Eds.), Actes de l'école chercheurs Inra en bioclimatologie, Le Croisic, 3-7 Avril 1995 Vol. Tome 1, Inra, ThivervalGrignon, France, 1996, pp. 351-369.

[2] Aries F., Modélisation surfacique d'un couvert végétal pour l'étude du rayonnement, Ph.D. thesis, École doctorale sciences pour l'ingénieur, Nantes, Nantes, 1997.

[3] Chelle M., Développement d'un modèle de radiosité mixte pour simuler la distribution du rayonnement dans les couverts végétaux, thèse, université de Rennes, Rennes, 1997.
[4] Curran P.J., Williamson H.D., Sample size for ground and remotely sensed data, Remote Sens. Environ. 20 (1986), 31-41.

[5] de Reyffie P., Edelin C., Francon J., Jaeger M., Puech M., Plant model faithful to botanical structure and development, Comput. Graphics 22 (1988) $151-158$.

[6] España M.L., Utilisation de maquettes dynamiques 3D pour suivre des cultures de maïs par télédétection, Ph.D. thesis, Université de Marne la Vallée, Marne la Vallée, France, 1997.

[7] España M., Baret F., Chelle M., Aries F., Andrieu B., A dynamic model of 3D architecture: application to the parameterisation of the clumpiness of the canopy, Agronomie 18 (1998) 609-626.

[8] Goel N.S., Rozehnal I., Thompson R.L., A computer graphics based model for scattering from objects of arbitrary shapes in the optical region, Remote Sens. Environ. 36 (1991) 73-104.

[9] Govaerts Y.M., Verstraete, M.M., Raytran: a Monte Carlo ray tracing model to compute light scattering in three dimensional heterogeneous media, IEEE Trans. Geosci. Remote Sens. 36 (1998) 493-505.

[10] Kuusk A., A multispectral canopy reflectance model, Remote Sens. Environ. 50 (1994) 75-82

[11] Lewis, P., Muller J.P., Botanical plant modelling for remote sensing simulations studies, in: Proc. IEEE Geoscience and Remote Sensing Symp. ('IGARSS'90'), Washington DC, 1991, pp. 1739-1782.

[12] Myneni R.B., Ross J., Photon-vegetation Interactions. Applications in Optical Remote Sensing and Plant Ecology, Springer Verlag, Berlin, Heidelberg, New York, 1991.

[13] Nilson T., Approximate analytical methods for calculating the reflection functions of leaf canopies in remote sensing applications, in: Myneni R., Ross J. (Eds.), Photon-vegetation Interactions, Springer, New York, USA, 1991, pp. 161-190.

[14] Pinty B., Verstraete M.M., On the design and validation of surface bidirectional reflectance and albedo models, Remote Sens. Environ. 41 (1992) 155-167.

[15] Verhoef W., Light scattering by leaf layers with application to canopy reflectance modeling: the SAIL model, Remote Sens. Environ. 16 (1984) 125-141.

[16] Verstraete M.M., Pinty B., Myneni R., Potential and limitations of information extraction on the terrestrial biosphere from satellite remote sensing, Remote Sens. Environ. 58 (1996) 201-214. 